\title{
Editors' note regarding citations of other work
}

\author{
David J. Cooper ${ }^{1}$ - Lata Gangadharan ${ }^{2}$. \\ Charles Noussair ${ }^{3}$
}

Experimental Economics expects authors to cite papers that are relevant to their work. This typically would include the seminal papers on a topic and papers that are directly related to the submitted paper's research design. For topics where a large number of papers exist, it is acceptable to cite a survey paper or only the most important papers in the literature. Authors are expected to cite directly relevant papers that come from other fields such as psychology, political science, accounting, finance, and marketing. While it is common for the referees and editors to suggest additional citations, it is ultimately the authors' responsibility to cite the relevant literature. Given the huge number of papers written in experimental economics, it is inevitable that authors will in some instances fail to cite relevant papers, but we expect authors to make a reasonable attempt to find and cite important related papers. If in doubt, it is better to have too many citations rather than too few.

The policy of Experimental Economics in the future is that we will not publish errata to correct a failure to cite previous work, except under unusual circumstances. In this regard, publication of this erratum should be viewed as a one-off and exceptional event. The policy of the journal will be to publish errata to an article only to correct important and critical errors in the description and reporting of the research presented in the article itself. As a general rule, the journal in the future will not publish errata to an article merely to point out omitted, inaccurate, or inappropriate, citations. Exceptions may be made to this rule, but we intend these to be very rare. Those authors who feel that they should have been cited or that were

Charles Noussair

cnoussair@email.arizona.edu

1 Florida State University, Tallahassee, Florida, USA

2 Monash University, Melbourne, Australia

3 The University of Arizona, Tucson, USA 
cited inaccurately in an article that we have published will have to use other means, such as posting notices on their own websites or contacting key researchers doing related work directly, to notify the research community of their concerns. 